\title{
La escuela: construcción de paz, potencial de memoria y socialización política con primera infancia
}

Schools: Peace-building, Memory Potential and Political Socialization with Early Childhood

A escola: construção de paz, potencial da memória e da socialização política com a primeira infância

\author{
Paola Andrea Carmona-Toro* (D) orcid.org/0000-0003-0164-6535 \\ María Camila Ospina-Alvarado** (iD) orcid.org/0000-0002-7271-151X
}

\section{Para citar este artículo}

Carmona-Toro, P. y Ospina-Alvarado, M. (2022). La escuela: construcción de paz, potencial de memoria y socialización política con primera infancia. Revista Colombiana de Educación, 1(84), 1-18.

https://doi.org/10.17227/rce.num84-10708. 


\section{Resumen}

Palabras clave: rol de la escuela; paz; socialización política; niñas y niños; potenciales

Keywords: school role; peace; political socialization; children; potentials

Palavras-chave: papel da escola; paz; socialização política; meninas e meninos; potenciais
La escuela se configura como un espacio para acciones de paz, vinculadas a las situaciones cotidianas que viven las niñas y los niños de la primera infancia; en este sentido, el objetivo general de este artículo de investigación apuntó a comprender el papel del potencial de la memoria y de la socialización política, para la reconfiguración de las formas de relacionamiento de las niñas y los niños para la construcción de paz en Centros de Desarrollo Infantil (CDI) e instituciones educativas en Bogotá, Apartadó y Manizales. Metodológicamente se trabajó desde un enfoque mixto: en términos cuantitativos se creó un instrumento ad hoc para medir, a través de múltiples variables e indicadores, la incidencia de estos potenciales; en términos cualitativos, se analizaron los relatos de las niñas y los niños. A modo de conclusión, la escuela es un espacio para la construcción de paz, desde el cual se tejen formas de relacionamiento con otras personas y con sus contextos. Los recuerdos permiten la reconfiguración del presente, y la escuela es uno de los escenarios para que dicha configuración se potencie. La paz se construye en la vida cotidiana en la escuela, donde las prácticas de participación y escucha posibilitan que las niñas y los niños sean constructores de la convivencia y el buen vivir.

\section{Abstract}

School is configured as the scenario for peaceful actions, linked to the daily situations that children in early childhood live. In this sense, the general objective of the research aimed to understand the role of the potential of memory and political socialization, for the reconfiguration of the ways of relating of children for peacebuilding in childcare centers and educational institutions in Bogotá, Apartadó and Manizales. Methodologically it worked from a mixed approach. A quantitative Ad Hoc instrument was created to measure the incidence of these potentials through multiple variables and indicators. At a qualitative level, the stories of the children were analyzed. In conclusion, the school is an environment for peacebuilding, in which the relationship between children and others, as well as their contexts are interwoven. Memories allow the reconfiguration of the present, and the school is one of the scenarios for this configuration to be enhanced. Peace is built-in to daily life in school, where the practices of participation and listening make it possible for children to be builders of coexistence and good living.

\section{Resumo}

A escola configura-se como um espaço de ações de paz, vinculadas às situações cotidianas vivenciadas por meninas e meninos da primeira infância; nesse sentido, o objetivo geral deste artigo de pesquisa apontou para compreender o papel do potencial da memória e da socialização política, para a reconfiguração das formas de relacionamento de meninas e meninos para a construção da paz nos Centros de Desenvolvimento Infantil (CDI) e instituições de ensino em Bogotá, Apartadó e Manizales. Metodologicamente trabalhou-se desde uma abordagem mista: em termos quantitativos, foi criado um instrumento ad hoc para medir, por meio de múltiplas variáveis e indicadores, a incidência desses potenciais; em termos qualitativos, foram analisadas as histórias de meninas e meninos. Concluindo, a escola é um espaço de construção da paz, a partir do qual se tecem as formas de relacionamento com as outras pessoas e com seus contextos. As memórias permitem a reconfiguração do presente, e a escola é um dos cenários para que essa configuração seja potencializada. A paz é construída na vida cotidiana da escola, onde as práticas de participação e escuta permitem que as meninas e meninos sejam construtores de convivência e bem viver. 


\section{Introducción}

En este artículo se condensan los resultados parciales de la investigación "Impactos de los potenciales humanos en los procesos de construcción de paz con participación de la primera infancia y sus agentes relacionales", adscrito al Cinde-Universidad de Manizales, y del proyecto "Memoria y socialización política de los niños y niñas de la primera infancia y sus familias para la territorialización de la paz", adscrito a la Universidad Católica Luis Amigó, en convenio con el Cinde-Universidad de Manizales; a partir de los cuales, se retoman los potenciales del desarrollo humano alternativo propuestos por el Programa Convidarte para la Paz (OspinaAlvarado et al., 2016) -afectivo, de vida y del cuerpo; ético, espiritual y de cuidado de la naturaleza; comunicativo, cognitivo y creativo para la transformación de conflictos; lúdico y de exploración; político; y el potencial de la memoria- como estrategias claves para la construcción de paz en la escuela.

Así, se piensa a la escuela como un espacio para la construcción de prácticas de paz, mediante el fortalecimiento del potencial de la memoria y la socialización política de las niñas y los niños de la primera infancia. Tanto la memoria como la socialización política se constituyen en herramientas para educar a los niños y las niñas en estas emergencias contemporáneas, que nos convocan a entenderlos como partícipes activos de los diferentes procesos de los cuales forman parte; teniendo en cuenta que tienen la capacidad para tomar decisiones; pensar en las otras personas, en el contexto que les rodea; conocer la incidencia de sus recuerdos y de su pasado en su vida presente, y son capaces de encontrar caminos de relacionamiento en su cotidianidad, por fuera de estructuras de violencia. Cabe mencionar que no pretendemos entregar responsabilidades de adultos a las niñas y los niños, ni delegar en ellas y ellos la trasformación de situaciones estructurales como la pobreza, la violencia barrial o social que involucran la necesaria acción estatal; por el contrario, entendemos que, desde las capacidades propias de su edad, pueden aportar a la transformación de algunas de sus prácticas diarias, específicamente de las que desarrollan en la escuela, como estar con otros en el aula y en los espacios comunes (patio, canchas deportivas, comedor escolar, biblioteca, entre otros).

Las niñas y los niños que hoy habitan las escuelas no deben ser leídos como sujetos pasivos ni como guiados solo por visiones adultocéntricas; en este sentido, el proceso de escolarización requiere de nuevas formas de abordaje, por ejemplo, la incorporación del pasado (de los recuerdos) como legado de las relaciones familiares, comunitarias y contextuales; la participación en los procesos de aprendizaje y en la toma de decisiones frente al estar con otros, y finalmente, a partir de la comprensión de que las niñas y los niños aportan a la construcción de paz desde su estar en la escuela y desde sus prácticas cotidianas con otros.

Así, se visibilizará un proceso local, a partir de los resultados obtenidos de tres Centros de Desarrollo Infantil (CDI) y dos instituciones educativas de las ciudades de Bogotá, Urabá y Manizales, con niñas y niños de la primera infancia. 


\section{Socialización política y construcción de paz}

La socialización política hace referencia a todos aquellos discursos y prácticas que configuran creencias, actitudes y formas de actuar, tanto a nivel individual como colectivo, promoviendo a los sujetos como agentes políticos capaces de incidir en la construcción y transformación de los sistemas políticos y normativos que los cobijan (Alvarado et al., 2012). Es la manera como las diferentes generaciones de una sociedad integran los contratos sociales y legitiman las normas y principios que establecen un orden social específico (Ospina-Alvarado et al., 2016). Cabe mencionar que la socialización política no se limita al cumplimiento de normas, por el contrario, posee una fuerza de producción y transformación social que emerge de la capacidad de agencia de los sujetos y las comunidades (Alvarado et al., 2012).

La socialización política implica la participación de agentes relacionales como la familia, los agentes educativos y la sociedad y la existencia de un lugar que la albergue, que permite su existencia, que haga posible su desarrollo y manifestación, es decir, los contextos en los que se encuentran inmersos los sujetos -la escuela, el hogar, entre otros-; entendiendo que estos, a su vez, están conformados por lo otro -la naturaleza, los objetos, los animales- y por el otro -seres humanos- que allí habitan (Ospina-Alvarado et al., 2016). En otras palabras, se tejen relaciones entre el contexto y los sujetos; para el caso de contextos de conflicto armado, donde se naturaliza la violencia y existen visiones deficitarias frente a los otros, la socialización política emerge como una forma de humanización (Ospina-Alvarado et al., 2016).

La construcción cultural del ser político es interpelada desde la socialización política como una forma activa de participar en estipulaciones políticas que anteceden y que ubica a los seres humanos, inicialmente, como sujetos pasivos que solo deben obedecer.

Por otro lado, como posibilidad para la construcción de paz, en 2016 en Colombia se firma el acuerdo final de paz con la guerrilla de las FARC-EP, este versa sobre la necesidad de una paz estable y duradera desarrollada a través de cinco puntos fundamentales, reforma rural integral, participación política, fin del conflicto, solución final al problema de las drogas y víctimas (Mesa de Negociación, 2017); aspectos estructurales y propios del conflicto armado colombiano. Sin embargo, la construcción de paz en Colombia y en otros países del Cono Sur trasciende estos cinco puntos y da cuenta de otras maneras desde las cuales, en los territorios, se está pensando la paz y cómo construirla de forma comunitaria, barrial, entre otras. Como lo proponen Salazar Castilla et al. (2018), para hablar de paz es indispensable reconocer la existencia de múltiples iniciativas tendientes a su construcción, como las acciones, las investigaciones y pedagogías, los emprendimientos y las políticas públicas.

Los espacios de construcción de paz, de tejer con las otras personas en torno a las prácticas que requiere cada territorio o contexto, deben estar acompañados de una cultura de paz, es decir, de la generación de prácticas de convivencia, tolerancia, procesos participativos, nuevas formas de relacionamiento, cambio de paradigmas, entre otras (Le Blanc, 2016), que permitan la construcción y la instauración de prácticas de construcción de paz. La escuela, se configura como uno de estos espacios para potenciar prácticas de construcción de paz, que obedezcan a las necesidades del contexto. La Oficina del Alto Comisionado para la Paz y la Organización Internacional para las Migraciones (OIM) (2015) ponen en voz de las niñas y los niños el significado de la paz, quienes la enuncian como: un país sin muertos, el cuidado de la naturaleza, que no existan conflictos en la comunidad, que exista respeto, entre otras formas de comprensión cercanas a la realidad que viven las niñas y niños en los contextos que habitan. 


\section{Memoria}

Para Ospina-Alvarado (2018), las niñas y los niños son seres con potencia y con capacidad de agencia; en la primera se encuentra la memoria, como potencial del desarrollo humano, desde el cual son capaces de resignificar lo ocurrido a través de sus aprendizajes y de asumir un rol activo en la construcción, tanto del presente como del futuro. La memoria es la capacidad que tienen los seres humanos de ir al pasado, traer un recuerdo al presente de algo que fue acontecimiento (Ricoeur, 2000); es una forma de hacer presente una ausencia (Ricoeur, 2013); es un acontecimiento que se teje con el pasado (Reyes Mate, 2008), en el que todas las personas son sujetos de memoria y generan sentidos identitarios y sociales en torno al pasado (Torres Carrillo, 2006; Jelin, 2002); que a su vez, se construyen con otros a través de marcos sociales (Halbwash, 2004).

La memoria es la capacidad de invocar y convocar, es la clave de todo lo que somos y lo que seremos (I diego, 2013) y su existencia se posibilita en espacios como la escuela, a partir de fundamentos epistemológicos, postura ética, responsabilidad política y académica (Torres, 2016); se constituye en un dispositivo de trabajo pedagógico (Jiménez Becerra et al., 2012), que, a su vez, permite innovar en las formas de enseñanza en la escuela, como posibilidad de problematizar lo que acontece a nivel familiar, comunitario, barrial y territorial; es el caso de la cultura política (Herrera y Pertuz, 2016), que no es posible sin el conocimiento del pasado, de lo ocurrido, por ejemplo, en contextos de violencia donde se propende por la reconciliación mediante memorias intergeneracionales.

Los recuerdos son la manifestación de hacer memoria, del acto de recordar; que se puede realizar desde tres grandes líneas (Betancourt, 2006); la memoria histórica, que da cuenta de sucesos específicos, de una época, un contexto y un acontecimiento, es la que se reconstruye a partir de datos históricos narrados en el presente, pero que pertenecen al pasado. La memoria colectiva da cuenta de los recuerdos de las experiencias o acontecimientos que tiene un grupo o una comunidad y que suele transmitirse a otra generación de individuos. La memoria individual es aquella que no puede ser experienciada o vivida por otros, pero que se convierte en la base de la memoria colectiva, como posibilidad de diálogo entre los recuerdos que los individuos tienen y que se resignifican colectivamente.

\section{Metodología}

El estudio realizado se basó epistémica y metodológicamente en la hermenéutica ontológica política o hermenéutica ontológica performativa que busca, a la vez, comprender y fortalecer procesos de transformación (Alvarado et al., 2014; Ospina-Alvarado et al., 2016), desde la cual, las niñas y los niños ponen sus voces, acciones y vivencias de la cotidianidad, para permitirnos comprender nuevas formas en las que se configura para ellos el estar en la escuela. Desde esta perspectiva, se realizaron talleres lúdico-pedagógicos vinculados a su mundo simbólico (creencias, imaginarios, significados que otorgan a vivencias); su mundo físico (espacios de estar con otros y elementos que configuran el mundo objetual), y finalmente el mundo social (formas de interacción con otros, compañeros del colegio, docentes, administrativos, familia).

El desarrollo metodológico se estructuró desde dos componentes: uno educativo, en el cual se despliegan los potenciales del desarrollo humano para la construcción de paz y un 
componente investigativo, que permitió dar cuenta de la memoria y la socialización política como potenciales que configuran algunas formas alternativas frente a aquellas tradicionales de comprender la escuela (como espacio solo de transmisión de conocimientos). El potencial de la memoria y el político es valorado desde tres dimensiones: la relación consigo mismo, la relación con los otros y la relación con lo otro.

\section{Caracterización de la muestra}

Se presentará el caso de tres CDI y dos instituciones educativas de Soacha (Bogotá), Urabá y Manizales, en los que se contó con 119 casos válidos. En cuanto a las edades, 75 niñas y niños tenían 4 años $(63,0 \%) ; 36$ tenían 5 años (30,3\%) y 8 tenían 6 años $(6,7 \%) ; 43$ eran de sexo masculino $(36,1 \%)$ y 76 de sexo femenino $(63,9 \%)$.

\section{Procedimiento}

Se trabajó en cinco momentos:

- Primer momento. Etapa de diseño, por parte del equipo de investigación, de los instrumentos para trabajo con los niños y las niñas. Se creó un instrumento ad hoc, para medir a través de múltiples variables e indicadores, la incidencia de estos potenciales. La variable memoria y la variable socialización política tuvieron tres dimensiones (consigo mismo, con el otro y con lo otro), cada una con tres indicadores latencia (refiere la existencia del potencial, pero sin que se haya puesto en práctica), existencia (cuando existen los potenciales, pero son vividos parcialmente en sus prácticas cotidianas) y apropiación (cuando los potenciales se viven en pleno). El diligenciamiento del instrumento se realizó a través de observación de la forma como las niñas y los niños respondían a diferentes situaciones. También se recurrió al diario de campo para consignar las observaciones del trabajo y los relatos de las niñas y los niños, durante las actividades.

- Segundo momento. Etapa de aplicación de los instrumentos de manera individual y colectiva, mediante un pretest.

\section{Tabla 1}

Breve descripción talleres pretest y postest

\begin{tabular}{lll}
\hline \multicolumn{1}{c}{ Taller } & \multicolumn{1}{c}{ Dimensiones } & \multicolumn{1}{c}{ Breve descripción de la actividad } \\
$\begin{array}{l}\text { Potencial político: La casa } \\
\text { en común }\end{array}$ & $\begin{array}{l}\text { Relación consigo } \\
\text { mismo }\end{array}$ & $\begin{array}{l}\text { Consiste en construir colectivamente una } \\
\text { casa con cajas de cartón, para lo cual, cada } \\
\text { niño y niña deberá elegir una de las cajas } \\
\text { para aportar a su construcción. Una vez }\end{array}$ \\
& seleccionada, se formula la pregunta. ¿Por \\
& qué elegiste esa caja y qué parte de la casa \\
& podrías construir con ella?
\end{tabular}




\begin{tabular}{|c|c|c|}
\hline & $\begin{array}{l}\text { Relación con el } \\
\text { otro }\end{array}$ & $\begin{array}{l}\text { Se les indicará a los niños y niñas que el } \\
\text { propósito de la actividad es realizar una } \\
\text { casa donde quepan todos los amiguitos y } \\
\text { todas las amiguitas. Los niños y niñas } \\
\text { tendrán que conversar y ponerse de } \\
\text { acuerdo para construir colectivamente la } \\
\text { casa. }\end{array}$ \\
\hline & $\begin{array}{l}\text { Relación con lo } \\
\text { otro }\end{array}$ & $\begin{array}{l}\text { Una vez construida la casa, se narra una } \\
\text { corta historia a los niños y niñas frente al } \\
\text { problema que se tiene; ahora la casa la } \\
\text { quieren tumbar para hacer otra } \\
\text { construcción, y los amiguitos y las amiguitas } \\
\text { que vivían ahí no saben qué hacer. (Se } \\
\text { puede hacer uso del cuento los tres } \\
\text { cerditos). Se organizarán grupos para } \\
\text { representar cómo continúa la historia, } \\
\text { respondiendo la siguiente pregunta: "¿Qué } \\
\text { vamos a hacer entre todos y todas para que } \\
\text { esto no ocurra?". }\end{array}$ \\
\hline \multirow[t]{3}{*}{$\begin{array}{l}\text { Potencial de la memoria: } \\
\text { Viaje al pasado }\end{array}$} & $\begin{array}{l}\text { Relación consigo } \\
\text { mismo }\end{array}$ & $\begin{array}{l}\text { Se cantará una ronda y, cuando la máquina } \\
\text { del tiempo pare, se dará la instrucción a los } \\
\text { niños y niñas de que se ha vuelto al pasado, } \\
\text { ellos y ellas tendrán materiales para pintar } \\
\text { cómo se recuerdan en el pasado. }\end{array}$ \\
\hline & $\begin{array}{l}\text { Relación con el } \\
\text { otro }\end{array}$ & $\begin{array}{l}\text { El maquinista, una vez realice su ronda, les } \\
\text { dirá a los niños y niñas que vamos a } \\
\text { recordar situaciones o momentos vividos } \\
\text { (puede ser en su hogar, escuela, comunidad } \\
\text { o demás espacios). }\end{array}$ \\
\hline & $\begin{array}{l}\text { Relación con el } \\
\text { otro }\end{array}$ & $\begin{array}{l}\text { Ahora, el maquinista, una vez cante la } \\
\text { ronda, dará la instrucción de que los niños y } \\
\text { niñas deberán hacer un dibujo de un lugar } \\
\text { que recuerden y explicar el motivo de su } \\
\text { elección. Se realizan mapas (cartografías). }\end{array}$ \\
\hline
\end{tabular}

Fuente: Ios talleres hacen parte de la propuesta Convidarte para la paz (Ospina-Alvarado et al., 2020).

- Tercer momento. aplicación de la propuesta educativa y trabajo conjunto de revisión de la información recolectada para identificar el impacto que estaban teniendo los talleres; en los casos que fue necesario, hubo adaptaciones a dinámicas instituciones, por ejemplo, el tiempo de trabajo con las niñas y los niños.

- Cuarto momento. Posterior a la propuesta educativa, se aplica un postest de los instrumentos, que permitió hacer seguimiento al trabajo.

- Quinto momento. Análisis de la información obtenida en torno al potencial de la memoria y el potencial de socialización política y relación que ambos guardan con la escuela como espacio para la construcción de paz. 


\section{Resultados}

Con relación al potencial de la memoria, la investigación permitió identificar que las niñas y los niños sí hacían memoria de sus vivencias; es decir, tenían la capacidad para evocar y recordar diferentes acontecimientos de su vida familiar, comunitaria, social y educativa, pero no lo habían desplegado plenamente; por lo cual, el potencial de la memoria se encontraba en el indicador de existencia. Al observar las dimensiones, se puedo establecer que la dimensión en la que este potencial estaba más alto era en la de relación con los otros, que inició con una media de 77,55, después de los talleres, y en la aplicación del postest, la media cambio a 86,12, como se visualiza en la tabla 2, pasando del indicador de existencia al indicador o estado de apropiación.

Tabla 2

Potencial de la memoria. Relación dimensiones media pretest/media postest. Indicadores

\begin{tabular}{lll}
\hline Dimensiones & $\begin{array}{l}\text { Media inicial } \\
\text { pretest }\end{array}$ & Media postest \\
Consigo mismo & 71,8 & 93,03 \\
& $\begin{array}{l}\text { Indicador } \\
\text { existencia }\end{array}$ & Indicador apropiación \\
Con el otro & 77,55 & \\
& $\begin{array}{l}\text { Indicador } \\
\text { existencia }\end{array}$ & Indicador apropiación \\
& & \\
Con lo otro & 72,24 & 86,94 \\
& $\begin{array}{l}\text { Indicador } \\
\text { existencia }\end{array}$ & Indicador apropiación \\
\hline
\end{tabular}

Fuente: elaboración propia.

Este resultado cuantitativo del peso relacional en las memorias coincide con lo encontrado a nivel cualitativo, al identificar que los recuerdos de las relaciones eran también los más recurrentes en las historias de los niños y las niñas, aquellos que se refieren como acontecimientos en sus vidas. También este hallazgo coincide con la importancia de las relaciones que otros estudios han encontrado frente a la primera infancia, los cuales han enfatizado en la centralidad de las relaciones familiares (Fujimoto, 2011; Marchesi, 2011; Peralta, 2011; Ramírez, 2011; Richter et al., 2018; Romero y Castañeda, 2009; Sarlé y Arnaiz, 2011; Zhang et al., 2018), pero sin referir su presencia en las memorias de los niños y las niñas. Sin embargo, aun cuando las relaciones familiares han sido aquellas identificadas como las más significativas para los niños y las niñas en sus primeros años de vida, llama la atención que las relaciones en la escuela son aquellas que están más presentes en sus relatos de 
remembranzas, hallazgo que una vez más coincide con los resultados cuantitativos referidos previamente. Cabe señalar que el presente estudio se desarrolló en el contexto de los CDI y las instituciones educativas, lo que pudo haber llevado a que sus recuerdos evocaran el ambiente escolar.

Las memorias de las relaciones con los otros en la escuela se focalizan en la manera como las niñas y los niños conciben las relaciones con sus compañeros y compañeras, a través de recuerdos de vivencias como: aquello que más disfrutaban era jugar con sus compañeros en la cancha o en el patio, cuando la profesora les enseñó una canción o cuando disfrutaban de las actividades que les hacían para celebrar días especiales de los niños; estos recuerdos suelen ser alegres y con la añoranza de que los acontecimientos se prolonguen de la misma forma en el presente. También se presentaron recuerdos relacionados con situaciones que generaron tristeza, como verse inmensos en peleas con los compañeros o cuando se sintieron rechazados por compañeros o profesores.

Al permanecer parte de su vida en la escuela, muchos de sus recuerdos se relacionan con este espacio vital: jugar, estar con los compañeros, cuando los profesores los tratan bien, cuando hacen actividades de las que ellas y ellos han participado, cuando los premiaban por portarse bien en la clase, entre otros. Frente a la pregunta por las acciones necesarias para estar en paz en la escuela cuando se han presentado situaciones conflictivas, las niñas y los niños refieren como posibilidad de estar en paz y construir paz con los compañeros de la escuela, aprender valores. A modo de ejemplo, se presenta uno de los trabajos realizados por las niñas y los niños (figura 1 ).

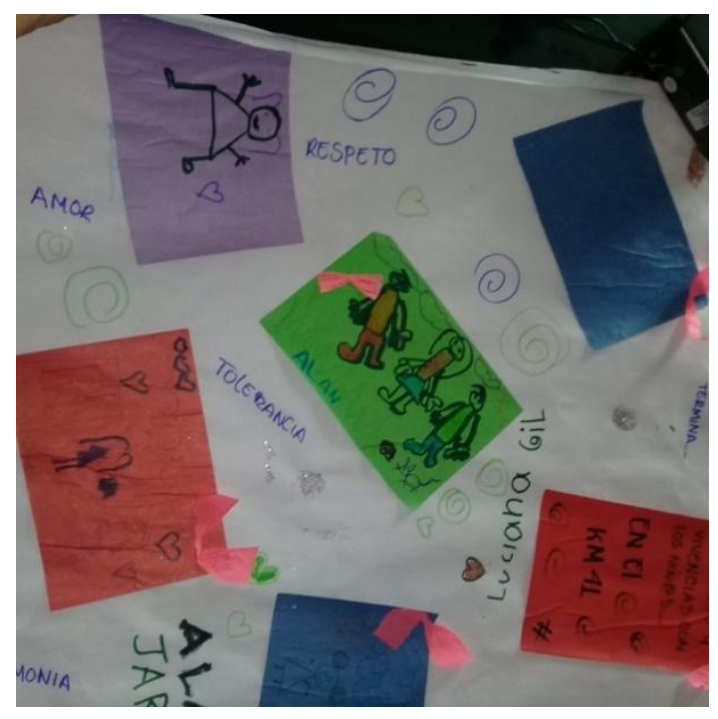

Figura 1. Valores para la paz

Fuente: fotografía tomada en el marco del proyecto, con previa firma de consentimiento informado para su uso.

La escuela se consolida para las niñas y los niños como un espacio para el aprendizaje de valores que permitan la convivencia y las prácticas de paz con todas personas que forman 
parte de la institución educativa o del CDI; los valores más enunciados fueron la tolerancia, el amor y el respeto. La escuela también se convierte en un espacio referencial y de los recuerdos; para ejemplificar, se presenta una de las cartografías realizadas con las niñas y los niños durante el taller de memoria (figura 2), en la que ellas y ellos ponen en el centro de sus recuerdos la institución educativa a la cual pertenecen; la escuela, al igual que la relación con el contexto que les rodea es frecuente en las diferentes cartografías que se realizaron.

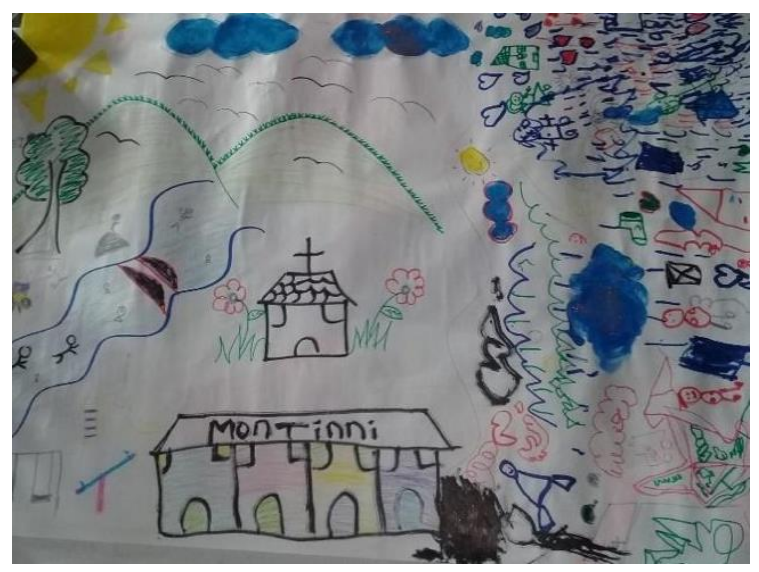

Figura 2. Cartografía de la memoria

Fuente: fotografía tomada en el marco del proyecto, con previa firma de consentimiento informado para su uso.

En este sentido, los recuerdos de las relaciones que tienen las niñas y los niños con sus compañeros, compañeras, docentes y demás personas que forman parte de la escuela, inciden en sus maneras de entender el presente e incluso su proyección a futuro. Cuando el recuerdo es alegre, acompañado de prácticas de paz, esto repercute en cómo viven su estar, en el presente, desde la convivencia pacífica; en tanto que, cuando los recuerdos se enmarcan en el dolor o en prácticas desde las cuales se sienten violentados, sus acciones en el presente suelen estar enmarcadas en el distanciamiento de prácticas colectivas como el juego o de la interacción con otros compañeros de clase o de la escuela.

Seguida a la dimensión de relación con los otros, se encuentra la dimensión de relación con lo otro, cuya media inicial fue de 72,24 , y posterior a la realización de los talleres, se tuvo en el postest una media de 86,94 , como se visualiza en la tabla 3; al igual que en la dimensión anterior, se pasa de un estado o indicador de existencia a un estado o indicador de apropiación. Esta dimensión hace referencia al ámbito objetual de la memoria, en el cual las niñas y los niños, como se evidencia en la aproximación cualitativa del estudio, tienen recuerdos que se relacionan con aquello que les rodea, como: objetos, juguetes, fotos, vestidos, elementos de la naturaleza y otras formas de vida -arboles, espacios naturales, ríos, plantas- y principalmente aluden a recuerdos muy felices que han compartido con sus mascotas, desde el día en que se las regalaron, hasta los diferentes acontecimientos que viven como verlas crecer y jugar con ellas. Lo otro se configura imbricado a los recuerdos de los otros, como aparece en la figura 2, los seres humanos; tienen vivencias en el río, en medio de las montañas 
y acompañados por las nubes, el sol, la luz, entre otras formas sensibles de la memoria. Esta memoria objetual que se configura propicia recuerdos que llegan en forma de imágenes, con colores y formas reconocidas; aspecto muy cercano a la propuesta de Ricoeur, para quien los recuerdos son traídos al presente a través de una representación o imagen (2000).

Tabla 3

Potencial político. Relación dimensiones media pretest/media postest. Indicadores

\begin{tabular}{lll}
\hline Dimensiones & $\begin{array}{l}\text { Media } \\
\text { inicial } \\
\text { pretest }\end{array}$ & $\begin{array}{l}\text { Media } \\
\text { postest }\end{array}$ \\
$\begin{array}{lll}\text { Consigo } \\
\text { mismo }\end{array}$ & $\begin{array}{l}70,57 \\
\text { Indicador } \\
\text { existencia }\end{array}$ & $\begin{array}{l}91,32 \\
\text { Indicador } \\
\text { apropiación }\end{array}$ \\
& & \\
Con el otro & 70,08 & 75,47 \\
& $\begin{array}{l}\text { Indicador } \\
\text { existencia }\end{array}$ & $\begin{array}{l}\text { Indicador } \\
\text { existencia }\end{array}$ \\
& & \\
& 64,53 & 73,21 \\
Con lo otro & Indicador & Indicador \\
latencia & \\
\hline
\end{tabular}

Fuente: elaboración propia.

En el caso de la escuela, los espacios son importantes, porque cada uno de ellos dota de sentido y de simbolismos los lugares del recuerdo; así, se encontró que para la mayoría de las niñas y los niños los lugares de los recuerdos felices están en la cancha y los espacios comunes de juego; los entornos de amor y acompañamiento los recuerdan en el aula de clase, porque está la profesora que los quiere. Sin embargo, también se encontraron recuerdos, donde los espacios comunes son de tristeza, porque es lugar en el que se materializa la discriminación hacia algunas niñas y algunos niños por parte de sus compañeros y compañeras.

Finalmente, la dimensión que inició más baja fue la relación consigo mismo, con una media en el pretest de 71,8 ; no obstante, fue la dimensión que más se incrementó posterior al trabajo con los talleres, pasando en el postest a una media de 93,03. Esta refiere a aquellos recuerdos que las niñas y los niños tienen sobre sí mismos; es decir, de su vida. Aquí siempre apareció, en la dimensión cualitativa del estudio, la memoria social, la que se construye con otros; por un lado, emergió como memoria generacional, en la que sus recuerdos obedecen a acontecimientos que les han contado sus familiares, docentes o vecinos. O como memoria social, en la que los recuerdos se narran siempre en relación con los otros y con lo otro. Por esta razón, los recuerdos de sí mismos aún no se veían consolidados al inicio de la investigación, pero, posteriormente, se pudo establecer que realizar talleres de activación de la memoria individual contribuye a que las niñas y los niños traten de pensarse a sí mismos, por ejemplo, desde su corporeidad y sus cualidades. 
Por otro lado, con relación a la socialización política, se tuvo en cuenta un elemento que contribuye a su configuración y que es el potencial político de las niñas y los niños. A partir de las tres dimensiones, se encontró que la dimensión de relación consigo mismo tuvo como media inicial 70,57, y después de los talleres, en la aplicación del postest, la media fue de 91,32 , esto significa que las niñas y los niños iniciaron en indicador de existencia y posterior a los talleres alcanzaron el nivel de apropiación del potencial. La relación consigo mismos emergió como la posibilidad que tuvieron de elegir por sí mismos y tomar decisiones frente a situaciones que se les presentaron; cada una y cada uno, a partir de los talleres, se asume como agente que decide, que opina, que manifiesta su inconformidad ante lo que no le gusta y que, desde su ser de niña y niño, considera justo o injusto lo que se evidenció a partir de la observación y de sus relatos. La posibilidad de elegir y pronunciarse ante los otros se acercó a un ejercicio político de lograr pese la diferencia, no de generar acciones violentas, sino de diálogo. Fue común encontrar que las niñas y los niños más silenciosos se inquietaban por tomar la palabra y ceder el turno a otros compañeros en la elección de los materiales y en el momento de comunicar el porqué de su elección.

Sin embargo, al pasar a la relación con los otros, esta inicia con una media de 70,08 y alcanza posterior a la aplicación de los talleres una media de 75,47, es decir, en esta dimensión no se presentó mayor variación; las niñas y los niños continuaron en indicador de existencia, teniendo el potencial, pero sin un pleno despliegue de este. La relación con los otros permaneció en cierta medida igual; como se identificó a través de la observación y de los relatos de los niños y las niñas cuando se pasaba de la dimensión individual a la dimensión de construir con otros, la dinámica cambiaba mucho, se tornaba más imitativa o de espera; en ocasiones se presentaron diferencias y malestar para construir juntos, para escuchar al otro o para participar de las elecciones que implicaban construir juntos. La figura 3 muestra uno de los ejercicios colectivos con los que se logró la participación de todos en la construcción de una casa, allí cada una y cada uno debía aportar y dar cuenta de por qué elegía contribuir en la elaboración de esa parte de la casa; pese a que el ejercicio generó interés hacia la construcción, los lazos relacionales no fueron tan claros y el interés continuaba siendo más individual que colectivo. También fue común escuchar discursos instaurados y hegemónicos en torno al rol de las mujeres en la elaboración de la casa o la incapacidad de los más silenciosos para aportar.

La presencia de discursos hegemónicos se constituye en un reto para la escuela que puede ser asumido, desde la socialización política, como forma de desmitificar en las niñas y los niños la instauración de roles, por ejemplo, vinculados a las capacidades que tanto las mujeres como los hombres tienen a nivel social; así como para contribuir en la comprensión que ellas y ellos pueden tener de la política como una forma de vivir juntos, mediante el diálogo con las demás personas y el involucramiento en las construcciones colectivas, reflejados en acciones tan pequeñas como elaborar una casa; ya que la capacidad de elegir y pronunciarse como se evidencia en la primera dimensión, debe estar acompañada del estar con otros. 


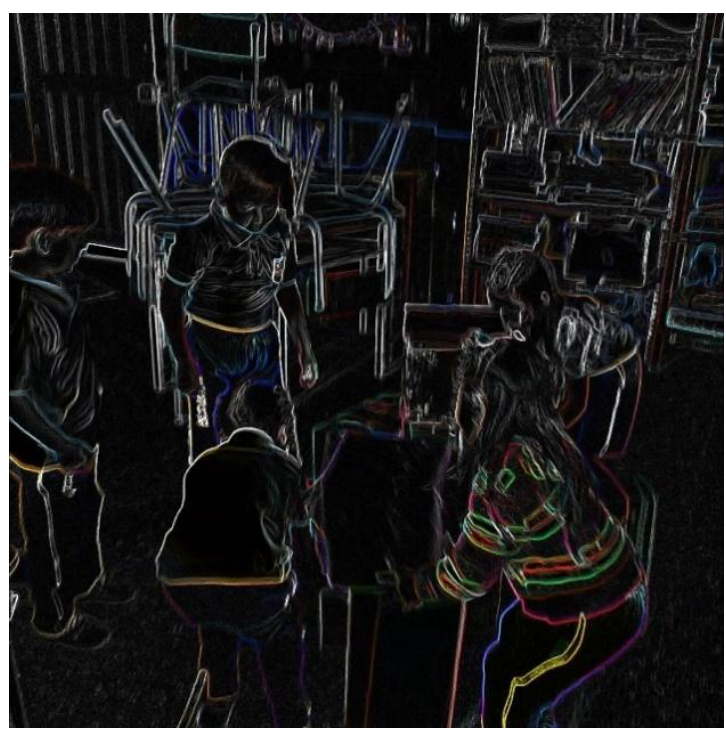

Figura 3. Construcción de la casa común

Fuente: fotografía tomada en el marco del proyecto, con previa firma de consentimiento informado para su uso.

Finalmente, la relación con lo otro permitió evidenciar que la media inicial fue de 64,53 y la media después de los talleres y en la aplicación del postest fue de 73,21 ; esto muestra que la relación de las niñas y los niños con los otros parte de indicador de latencia, es decir, existe el potencial político, pero no se había puesto en marcha; posterior a la aplicación, se llega al indicador de existencia, en el que se presenta cierto despliegue del potencial; pese a ello, no se alcanzó el nivel de apropiación frente al cuidado de aquello que colectivamente se construye y que permite vivir juntos, en paz con los otros y con lo otro. Este hallazgo se mantuvo también a nivel cualitativo en los relatos de los niños y las niñas, y en la observación desarrollada tanto por el equipo de investigación como del grupo de docentes y agentes educativas en su calidad de coinvestigadoras del proceso, al identificar que frente a la acción colectiva como práctica de socialización política había presencia, mas no centralidad, de los relatos acerca del cuidado de lo otro, por ejemplo del cuidado del medio ambiente como una acción colectiva con participación activa de los niños y las niñas en sus primeros años de vida.

Teniendo presente el potencial de la memoria y la socialización política relacionada con el potencial político y el trabajo que se puede hacer desde la escuela a partir de los recuerdos y hacia la paz, es necesario comenzar por decir que la paz en los CDI o las escuelas donde se trabaje con niñas y niños en primera infancia, no es una construcción institucionalizada para la transformación de asuntos estructurales, como por ejemplo, sí lo busca ser el acuerdo final de paz, firmado en 2016 en Colombia -desarrollo agrario integral, participación política, solución al problema de las drogas ilícitas, acuerdo sobre las víctimas, finalización del conflicto- (Mesa de Negociación, 2017). La paz en las escuelas y para las niñas y los niños de la primera infancia se refiere a sus maneras de relacionamiento en la cotidianidad consigo mismos, con los otros y con lo otro y con las situaciones que les afectan y, que desde un lente adultocéntrico, podrían pasar desapercibidas, como son: que un compañero o una compañera tome sus pertenencias sin permiso, que le escondan el morral, que algún compañero o alguna 
compañera se coma el algo o la merienda de otra persona, que la docente les regañe, entre otras situaciones, desde las cuales es muy importante comenzar a gestar formas de transformación pacífica de los conflictos.

\section{Conclusiones}

La escuela es un espacio para configurar maneras de estar con otras personas y aprender con ellas desde acciones pequeñas en el aula de clase y espacios comunes que habitan las niñas y los niños; es decir, la escuela, como lugar donde pasan la mitad de su tiempo diario, incide en la configuración de acciones y discursos que desde la primera infancia comienzan a tejerse y que pueden ser a mediano y largo plazo beneficiosos o perjudiciales para la construcción de paz. También en la escuela se gestan creencias perjudiciales como la naturalización de las violencias o la reproducción de discursos excluyentes, como se identifica en los relatos de los niños y las niñas: "no hablo con ella por el color de piel", "porque huele mal", "porque viven en una parte de la ciudad diferente a la mía"; entre otras narrativas que evidencian discursos dominantes, que desde la escuela se pueden interpelar, al comenzar con acciones que aparentemente son pequeñas, como escuchar y preguntarles a las niñas y los niños las razones de sus creencias y la forma como ellas y ellos creen que podrían cambiar dichas situaciones. Pequeños gestos que a futuro tendrán grandes repercusiones en las formas de relacionamiento que las niñas y los niños vayan tejiendo con otros y con el contexto a medida que crezcan, al tener en cuenta que el mayor potencial se encuentra, como lo ha mostrado Heckman (2000), en los primeros años de vida.

La escuela, como espacio para potenciar la construcción de paz, tiene una herramienta fundamental que es la memoria, no la instrumental o de trabajo, sino la memoria de la vida, de los recuerdos de aquello que ha configurado a las niñas y los niños en sus primeros años frente a sus creencias, acciones y formas de estar en el presente; campo que aún no es muy explorado; y que, como lo plantea Lykes (2003), lleva a que con el silenciamiento de las memorias las nuevas generaciones construyan especulaciones frente a lo sucedido. Situación que se puede explicar, en gran medida, en la eliminación de la clase de historia en muchas instituciones educativas de Colombia, dejando sin espacio los recuerdos tanto alegres como de dolor que ellas y ellos tienen, y aquellas que tienen las comunidades de las que participan. Incluir en la reflexión las voces y los recuerdos de las niñas y los niños les puede aportar a los adultos, con la mediación de relaciones intergeneracionales, en la comprensión del sentido de aquello que evocan a modo de añoranza y que en el presente intentan volver a vivir o que les aleja de aquellas situaciones de dolor; a modo de ejemplo, preguntar a las niñas y los niños qué recuerdos de la escuela les hacen felices, qué hace que quieran permanecer con dichos recuerdos o que se quieran distanciar de estos.

Teniendo en cuenta la importancia de la familia en la primera infancia y de las articulaciones entre la familia y la escuela (Ospina-Alvarado, 2014), es fundamental también conocer, en el ámbito familiar, qué recuerdan y que ese sentir sea incorporado al trabajo en las escuelas de padres. De modo que los propios niños y las propias niñas puedan aprender de las experiencias vividas por sus familias. Porque conocer el pasado no es garantía de no repetición, pero es un paso importante para reconfigurar el presente y las posibilidades de acción futura, y la escuela es un escenario que necesariamente debe convocar a la transformación y no solo a la transmisión.

Para finalizar, es importante comprender que la paz no se teje en el campo polisémico de construcciones teóricas, ese puede ser uno de los caminos; pero que los lugares para 
albergarla y comenzar a hacer de ella una realidad alcanzable, tanto para los sujetos como para las comunidades, están en la vida diaria y cotidiana; tal es el caso de la escuela, donde las prácticas de participación y escucha contribuyen a la configuración de las niñas y los niños como constructores de la convivencia y el buen vivir, mediada por el acompañamiento de docentes, directivos, familias y comunidad. La comprensión en torno a la participación es posible en tanto se dé el acto per se de dejarles participar, transformar conflictos de manera creativa y de desaprender órdenes discursivos hegemónicos, que han prevalecido por mucho tiempo, por ejemplo, el que solo la mayoría de edad (18 años en Colombia) entrega el derecho a participar políticamente. Las prácticas diarias, cotidianas; desear otras actividades para aprender; hacer actividades; elegir lo que se desea hacer durante el descanso o en las horas de ocio en la escuela; elegir quién debe ser el compañero que iza bandera, son el primer ejercicio participativo de las niñas y los niños, desde el cual aprenderán tanto a ser sujetos de la política, como sujetos inmersos en lo político: sujetos políticos.

\section{Referencias}

Alvarado, S. V., Ospina-Alvarado, M. C. y García, C. M. (2012). La subjetividad política y la socialización política, desde las márgenes de la psicología política. Revista Latinoamericana de Ciencias Sociales, Niñez y Juventud, 10(1), 235-256.

Alvarado, S. V., Gómez, A., Ospina-Alvarado, M. C. y Ospina, H. F. (2014). La hermenéutica ontológica política o hermenéutica performativa: una propuesta epistémica y metodológica. Revista Nómadas, 40, 207-220.

Betancourt, D. (2006). Memoria individual, memoria colectiva y memoria histórica. Lo secreto y lo escondido en la narración y el recuerdo. En A. Jiménez Becerra y A. Torres Carrillo (comps.), La práctica investigativa en ciencias sociales (pp. 125-136). Fondo Editorial Universidad Pedagógica Nacional.

Fujimoto, G. (2011). El futuro de la educación iberoamericana: ¿Es la no escolarización una alternativa? En J. Palacios y E. Castañeda (coords.), La primera infancia (0-6 años) y su futuro (pp. 105-114). Organización de Estados Iberoamericanos para la Educación, la Ciencia y la Cultura (OEI).

Halbwash, M. (2004). La memoria colectiva. Prensas Universitarias de Zaragoza.

Heckman, J. (2000). Policies to foster human capital. Northwestern University.

Herrera, M. C. y Pertuz, C. (2016). Educación y políticas de la memoria sobre la historia reciente de América Latina. Revista Colombiana de Educación, (71), 79-108.

Jelin, E. (2002). De qué hablamos cuando hablamos de memoria. En Los trabajos de la memoria (pp. 17-38). Siglo XXI Editores.

Jiménez Becerra, A., Infante Acevedo, I. y Cortés, R. (2012). Escuela, memoria y conflicto en Colombia. Un ejercicio del estado del arte de la temática. Revista Colombiana de Educación, (62), 287-314.

I diego (17 de noviembre de 2013). Memorias de fuego-Eduardo Galeano [Archivo de video]. YouTube. https://www.youtube.com/watch?v=AC1hLEq5UAg 
Le Blanc, J. (2016). Cultura de paz y de reconciliación. En M. F. Vargas, A. Lóèz y J. A. Gómez (coords.), Construcción de desarrollo y paz: aprendizajes y recomendaciones desde los territorios (pp. 87-106). Redprodepaz.

Lykes, M. B. (2003). Una reinterpretación crítica del estrés pos- traumático desde una perspectiva comunitaria e intercultural. Psicología social y violencia política. Siglo XXI Editores.

Marchesi, A. (2011). Preámbulo. En J. Palacios y E. Castañeda (coords.), La primera infancia (0-6 años) y su futuro (pp. 7-10). Organización de Estados Iberoamericanos para la Educación, la Ciencia y la Cultura (oei).

Mesa de Negociación. (2017). Acuerdo final para la terminación del conflicto. Oficina Alto Comisionado para la Paz. https://www.unidadvictimas.gov.co/es/acuerdo-final-para-laterminacion-del-conflicto-y-la-construccion-de-una-paz-estable-y-duradera

Oficina del Alto Comisionado para la Paz y Organización Internacional para las Migraciones (OIM). (2015). Yo quiero paz, reconciliación y un país lleno de amor. https://repositoryoim.org/handle/20.500.11788/1267

Ospina-Alvarado, M. C. (2014). Las familias: sistemas interaccionales y construcciones relacionales, dialógicas, sociales, culturales e históricas. En Alvarado, S. V. y H. F. Ospina (eds.), Socialización política y configuración se subjetividades. Construcción social de niños y niñas y jóvenes como sujetos políticos (pp. 225-263). Siglo del Hombre Editores; Universidad de Manizales; Cinde.

Ospina-Alvarado, M. C. (2018). A manera de introducción: retomando la experiencia de la línea de investigación en construcción social del niño u la niña: familias y otros contextos relacionales. En M. Ospina-Alvarado (ed.), Construcción social de niñas y niños (pp. 3554). Universidad Pedagógica Nacional; Fundación Centro Internacional de Educación y Desarrollo Humano (Cinde), Universidad de Manizales.

Ospina-Alvarado, M. C., Alvarado, S. V., Cardona, L. M., Alvarado, L., Fajardo, M. A. y Carmona, P. A. (2020, en prensa). Convidarte para la Paz: niñas y niños de la primera infancia, familias y otros agentes relacionales. Cinde/Universidad de Manizlaes; Universidad Pedagógica Nacional.

Ospina-Alvarado, M. C., Alvarado, S. V. y Fajardo, M. A. (2016). Prácticas de transformación social e interculturalidad de niños y niñas en el contexto del conflicto armado colombiano: un abordaje desde la hermenéutica ontológica política. En V. Di Caudo, D. Llanos y M. C. Ospina-Alvarado (coords.), Interculturalidad y educación desde el sur: contextos, experiencias $y$ voces (pp. 269-294). UPS-GIEI/Clacso/Cinde. http://dspace.ups.edu.ec/bitstream/123456789/12775/1/Interculturalidad\%20y\%20educ acion.pdf

Ospina-Alvarado, M. C., Alvarado, S. V. y Serna, I. (2016). Socialización política y construcción social de subjetividades de niñas y niños en contextos de conflicto armado. En J. D. Castañeda Muñoz y Y. Gutiérrez Cabrera (comp.), Socialización política y construcción de subjetividades entre el devenir de la ética y la resistencia (pp. 89-109). Corporación Universitaria Minuto de Dios. Centro Regional Neiva.

Ospina-Alvarado, M. C., Loaiza, J. A. y Alvarado, S. V. (2016). Potenciales de paz. Revista Internacional Magisterio Educación y Pedagogía. La escuela y la paz, 81, 26-29.

Peralta, M. V. (2011). El futuro de la educación inicial iberoamericana: calidad desde la construcción de currículos en una perspectiva de posmodernidad. En J. Palacios y E. 
Castañeda (coords.), La primera infancia (0-6 años) y su futuro (pp. 83-90). Organización de Estados Iberoamericanos para la Educación, la Ciencia y la Cultura (OEI).

Ramírez, N. (2011). Primera infancia: una agenda pendiente de derechos. En J. Palacios y E. Castañeda (coords.), La primera infancia (0-6 años) y su futuro (pp. 63-72). Organización de Estados Iberoamericanos para la Educación, la Ciencia y la Cultura (OEI).

Reyes Mate, M. (2008). Tierra y huesos. Reflexiones sobre la historia, la memoria y la memoria histórica. En La herencia del olvido. Ensayos en torno a la razón compasiva (pp. 149-176). Errata Naturae editores.

Richter, L. M., Lye, S. J. y Proulx, K. (2018). Nurturing care for young children under conditions of fragility and conflict. New Directions for Child and Adolescent Development [Towards a More Peaceful World: The Promise of Early Child Development Programmes], 159, 13-26. doi: $10.1002 /$ cad.20232.

Ricoeur, P. (2000). La memoria, la historia y el olvido. Fondo de Cultura Económica de Argentina.

Ricoeur, P. (2013). Fase documental: la memoria archivada. En La memoria, la historia, el olvido (pp. 189-236). Fondo de Cultura Económica.

Romero, T. y Castañeda, E. (2009). El conflicto armado colombiano y la primera infancia. En A. Mejía (ed.), Colombia: huellas del conflicto en la primera infancia (pp. 31-53). Número Ediciones.

Salazar Castilla, M., Jaramillo Ferro, J., Aristizábal Ramírez, E. y Carmona Toro, P. (2018). Construcción territorial de la paz: polifonías y resistencias desde la ciudad de Manizales. Centro Editorial Universidad Católica de Manizales.

Sarlé, P. M. y Arnaiz, V. (2011). Juego y estética en educación infantil. En J. Palacios y E. Castañeda (coords.), La primera infancia (0-6 años) y su futuro (pp. 91-104). Organización de Estados Iberoamericanos para la Educación, la Ciencia y la Cultura (OEI).

Torres Carrillo, A. (2006). Subjetividad y sujeto: perspectivas para abordar lo social y lo educativo. Revista Colombiana de Educación, (50), 86-103.

Torres, L. (2016). Historia reciente en la escuela colombiana: acercamiento a las nociones de memoria, historia y conflicto. Revista Colombiana de Educación, (71), 165-185.

Zhang, N., Zhang, J., Gewirtz, A. H. y Piehler, T. F. (2018). Improving parental emotion socialization in military families: Results of a randomized controlled trial. Journal of Family Psychology, 32(8), 1046-1056. doi:10.1037/fam0000461. 